\title{
Assessing the Impact of Canopy Structure Simplification in Common Multilayer Models on Irradiance Absorption Estimates of Measured and Virtually Created Fagus sylvatica (L.) Stands
}

\author{
Dimitrios Biliouris ${ }^{1, *}$, Dimitry Van der Zande ${ }^{1}$, Willem W. Verstraeten ${ }^{1}$, Bart Muys ${ }^{2}$ \\ and Pol Coppin ${ }^{1}$
}

1 Department of Biosystems, Katholieke Universiteit Leuven, M3-BIORES, Willem de Croylaan 34, BE-3001 Leuven, Belgium; E-Mails: Dimitry.VanDerZande@biw.kuleuven.be (D.Z.);

Willem.Verstraeten@biw.kuleuven.be (W.V.); Pol.Coppin@biw.kuleuven.be (P.C.)

2 Department of Earth and Environmental Sciences, Katholieke Universiteit Leuven, Celestijnenlaan 200 E, BE-3001 Leuven, Belgium; E-Mail: bart.muys@ees.kuleuven.be

* Author to whom correspondence should be addressed; E-mail: Dimitrios.Biliouris@biw.kuleuven.be; Tel.: +32-16-329-739; Fax: +32-16-329-724.

Received: 16 September 2009; in revised form: 7 November 2009 / Accepted: 9 November 2009 / Published: 16 November 2009

\begin{abstract}
Multilayer canopy representations are the most common structural stand representations due to their simplicity. Implementation of recent advances in technology has allowed scientists to simulate geometrically explicit forest canopies. The effect of simplified representations of tree architecture (i.e., multilayer representations) of four Fagus sylvatica (L.) stands, each with different LAI, on the light absorption estimates was assessed in comparison with explicit 3D geometrical stands. The absorbed photosynthetic radiation at stand level was calculated. Subsequently, each geometrically explicit 3D stand was compared with three multilayer models representing horizontal, uniform, and planophile leaf angle distributions. The 3D stands were created either by in situ measured trees or by modelled trees generated with the AMAP plant growth software. The Physically Based Ray Tracer (PBRT) algorithm was used to simulate the irradiance absorbance of the detailed 3D architecture stands, while for the three multilayer representations, the probability of light interception was simulated by applying the Beer-Lambert's law. The irradiance inside the canopies was characterized as direct, diffuse and scattered irradiance. The irradiance absorbance of the stands was computed during
\end{abstract}


eight angular sun configurations ranging from $10^{\circ}$ (near nadir) up to $80^{\circ}$ sun zenith angles. Furthermore, a leaf stratification (the number and angular distribution of leaves per LAI layer inside a canopy) analysis between the 3D stands and the multilayer representations was performed, indicating the amount of irradiance each leaf is absorbing along with the percentage of sunny and shadow leaves inside the canopy. The results reveal that a multilayer representation of a stand, using a multilayer modelling approach, greatly overestimated the absorbed irradiance in an open canopy, while it provided a better approximation in the case of a closed canopy. Moreover, the actual stratification of leaves differed significantly between a multilayer representation and a 3D architecture canopy of the same LAI. The deviations in irradiance absorbance were caused by canopy structure, clumping and positioning of leaves. Although it was found that the use of canopy simplifications for modelling purposes in closed canopies is demonstrated as a valid option, special care should be taken when considering forest stands irradiance simulation for sparse canopies and particularly on higher sun zenith angles where the surrounding trees strongly affect the absorbed irradiance and results can highly deviate from the multilayer assumptions.

Keywords: tree architecture; canopy representation; irradiance absorption; PBRT ray tracing; AMAP model; Fagus sylvatica L.

\section{Introduction}

The light environment inside a forest canopy is a major factor influencing the surface energy balance, the canopy microclimate, as well as photosynthesis, and plant growth [1]. Studying the light distribution within a forest canopy is possible using radiation transfer models, provided that the stand architecture and leaf optical properties are adequately described [2]. These radiation transfer models simulate the effect of canopy geometry and structure on light interception and can link individual leaf light capture to gross photosynthetic assimilations [3-7]. However, because of the difficulties in acquiring detailed stand structure information, most of these models simplify the canopy structure, by using either big-leaf models [8-10] (single leaf assumption), or multi-layer models [11-14] (homogeneous horizontal and vertical layers containing the same amount of leaf area). These simplifications may lead to inaccurate simulations of light interception at the canopy and subsequently at the leaf level [15]. Multilayer canopy models require estimation of the reflection, transmittance, and scattering of radiation within each canopy layer, which requires certain assumptions regarding leaf orientation [16].

Canopy structural simplifications can be nullified by taking a more thorough approach which explicitly considers light interception by individual leaves. For example, using in situ data of tree architecture [17-18] or virtual environment plant growth algorithms that respect the plant's genetic code and reconstitutes tree morphology [19] such as the Atelier de Modelisation et d'Architecture des Plantes (AMAP) model [20]. These geometric explicit canopy architecture models are capable of describing the radiation regime within canopies using current advances in computer power and path 
tracing algorithms such as tRAYci [1] and the PBRT path tracer [21]. As a result they provide a more accurate calculation of light intercepted by particular elements of the canopy [22].

Considerable research has been carried out investigating big-leaf model behaviour, especially in simulating daily photosynthetic variations, in comparison with multilayer canopy representations $[14,23,24]$. Furthermore, multilayer models have been assessed in terms of different leaf angular assumptions and its affects on the simulation of the absorbed irradiance [25,26].

At the same time, scientists have incorporated three dimensional canopy elements [27,28] to assess light interception inside a canopy either by using cubic units that were either filled or empty with leaf area [5], or by using field measurements and Monte Carlo simulations of canopy gap fraction and LAI to examine the interaction of beam radiation with clumped forest canopies [25]. However, the contribution of explicit geometric canopy models compared to different multilayer canopy simplifications in calculating irradiance absorption and therefore surface radiation balance has not yet been thoroughly assessed and quantified.

The main objective of this paper is to determine the magnitude of difference on irradiance absorbance estimates between multilayer modelling approaches and 3D geometrically explicit canopy representations for stands with open and closed canopies, throughout the time span of an individual day. The procedure involves the development of an explicit 3D structure model that incorporates both leaf level detailed canopy description and leaf optical properties of four Fagus sylvatica (L.) stands with variable Leaf Area Index (LAI) values. Two stands were created using in situ tree architecture measurements while the other two were generated by the AMAP model. The detailed structure of the four stands was imported in the PBRT ray tracing software in order to compute light interception and absorbance at leaf level. Three concurrent multilayer representations with the same LAI as the 3D stands were constructed, each with different Leaf Angle Distributions (LAD), and their absorbed irradiance was compared with the 3D stands used as reference. Furthermore, the stratification of leaves was assessed in terms of Percentage of the Above Canopy Light (PACL) and percentage of sunny and shadow leaves among the 3D canopies and the multilayer models.

\section{Materials and Methods}

\subsection{Study Area}

Measurements were conducted in August and September 2004 in an even-aged nine year old Fagus sylvatica (L.) stand on a sand-loamy soil in Meerdaal forest, south of Leuven, Belgium (50 $48^{\prime} \mathrm{N}$, $4^{\circ} 20^{\prime} \mathrm{E}$ ). Trees were planted in a $2 \times 1.80 \mathrm{~m}$ spacing pattern and had an average height of $2.50 \mathrm{~m}$. Nine trees were manually measured in detail up to the leaf level. The surrounding stands were separated from the study area, so no significant limitation of irradiance due to shadow casting appeared, with the exception of early and late hours of the day, before 07:00 am and after 7:00 pm Central European Summer Time, respectively. 


\subsection{In-Situ Measurement of Tree Architecture}

To obtain the fundamental description of canopy geometry based on inclination, azimuth, surface area and location of the individual plant parts a specific measurement strategy was followed [29]. Tree architecture was determined manually in three steps: (i) measurement of the stem, (ii) branches, and (iii) leaves. For each of these steps the 'depth first order' strategy [30] was applied to make sure that none of the plant parts were omitted. Length, diameter, azimuth, and zenith values per node were measured, while the azimuth and zenith of the main nerve of each leaf was recorded. All leaves were measured in terms of azimuth and zenith of the main nerve with a compass and a protractor, while a tape and a calliper were used to measure the length and diameter of branches and stems, respectively. For each tree, 16 leaves were scanned from four branches evenly spaced between the top and the bottom of the trees. All the leaves were placed on millimetre graph paper and scanned using Corel scan software. For each leaf the surface area of the leaf blade, length of the major axis of the leaf blade (main nerve), length of the minor axis of the leaf blade (longest length perpendicular to major axis) and length of the petiole were extracted.

\subsection{Geometrically Explicit Stand Architecture Model}

\subsubsection{Stand architecture model based on in-situ measured trees}

Two virtual stands were created, with the same row orientation (facing $30^{\circ} \mathrm{NW}$ ) as in the field, from the measured data of the nine measured trees using the Matlab software (Figure 1a). To account for any possible edge effects, the nine measured trees were positioned in the centre of the stand, while 72 clones of those trees in eight blocks of the same grid dimensions are surrounding the central individuals. Shading from neighbour trees in a forest stand is a major constrain on the available light and this was incorporated as realistically as possible.

The trees were placed in a regular grid of $2.00 \mathrm{~m} \times 1.80 \mathrm{~m}$ covering an area of $32.87 \mathrm{~m}^{2}$ and comprised a total of 86,211 leaves and a LAI of 0.58. A second simulation with an LAI of 1.29 was obtained by reducing the spacing, between the same measured trees used above, down to $1.00 \mathrm{~m} \times 1.00 \mathrm{~m}$ grid and a corresponding stand area of $13.94 \mathrm{~m}^{2}$ (Figures 2a,b).

\subsubsection{Stand architecture model based on AMAP modelled trees}

To guarantee the availability of geometric grown forest stands with higher density and LAI and thus closed canopies, the AMAP plant growth model was used to generate nine 15 year old trees (Figure 1b).

These trees were used to simulate two stands of 81 trees each with LAI of 2.76 and 3.65 respectively (Figure 2c,d). The trees were placed in a regular grid of $4.00 \mathrm{~m} \times 4.00 \mathrm{~m}$, with the same row orientation as the field ones, while the higher LAI value of 3.65 was achieved by using an additional $25 \%$ number of leaves per tree for the second stand. The age of the AMAP trees was selected to mimic LAI values as encountered in natural conditions [31,32]. The AMAP model was successfully applied in different applications such as light penetration through tree crown [33], forest landscape visualization [34], and canopy parameters derivation from SAR data [35] and provides a 
quantitative approach of simulating plant architecture [20,36]. Information about the measured and simulated trees and stands are presented in Table 1.

Figure 1. Trees used for explicit simulation of the Fagus sylvatica (L.) canopy. Left (A), a 9 year old in situ measured tree; right (B) a 15 year old AMAP simulated tree.
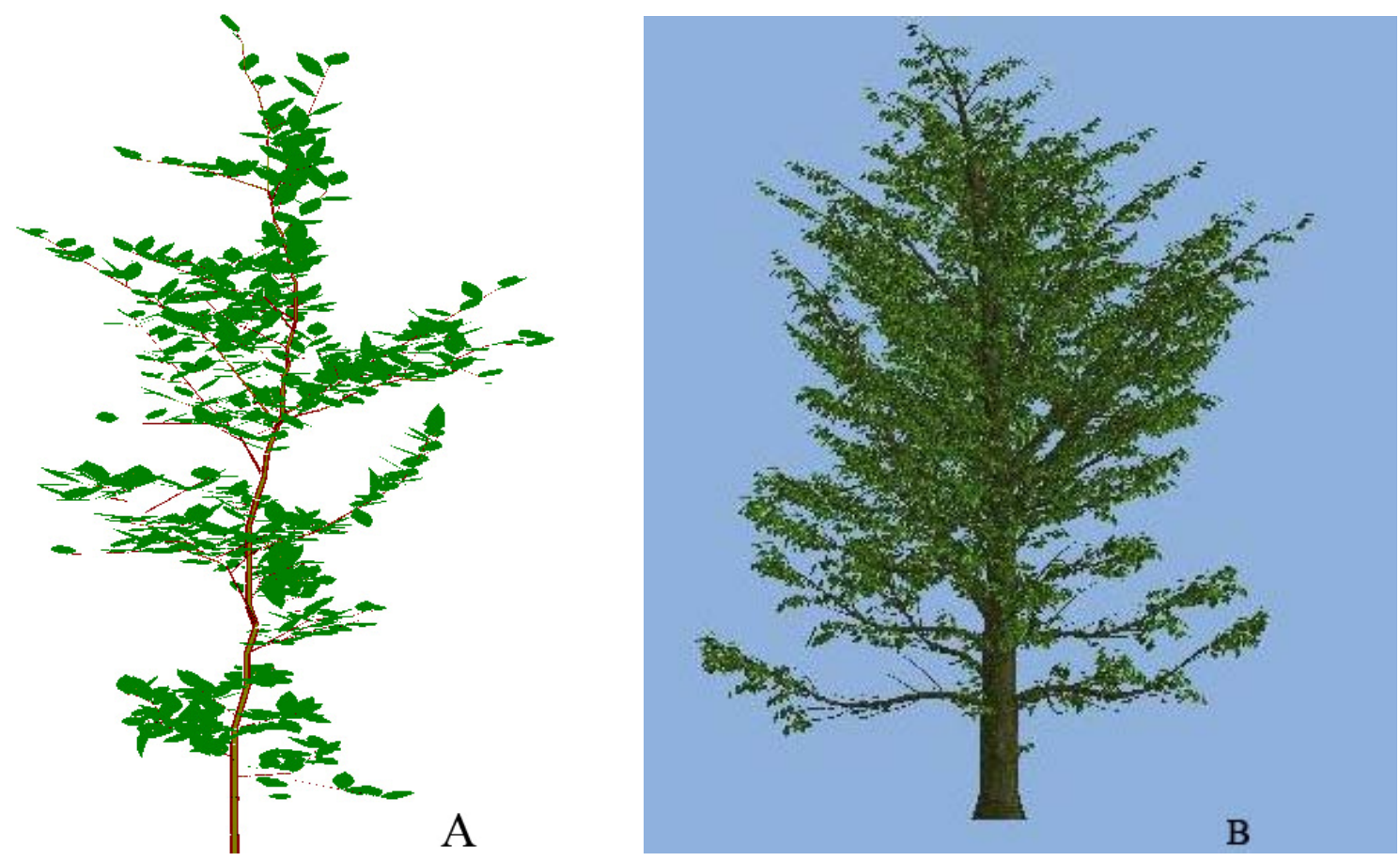

Table 1. Stand geometric information of the geometrically explicit 3D canopies.

\begin{tabular}{|c|c|c|c|c|c|c|}
\hline & LAI & $\begin{array}{c}\text { Mean Leaf Zenith } \\
\text { Angle }\left({ }^{\circ}\right)\end{array}$ & $\begin{array}{c}\text { Stand Area } \\
\left(\mathbf{m}^{2}\right)\end{array}$ & $\begin{array}{l}\text { Mean Crown } \\
\text { Diameter (m) }\end{array}$ & $\begin{array}{c}\text { Mean Leaf } \\
\text { Size }\left(\mathbf{m}^{2}\right) \\
\end{array}$ & $\begin{array}{l}\text { Mean Tree } \\
\text { Height (m) }\end{array}$ \\
\hline Stand A & 0.58 & 11.53 & 32.87 & 1.22 & 0.0018 & 2.50 \\
\hline Stand B & 1.29 & 11.53 & 13.94 & 1.22 & 0.0018 & 2.50 \\
\hline Stand C & 2.76 & 18.24 & 152.41 & 4.54 & 0.0013 & 9.75 \\
\hline Stand D & 3.65 & 18.92 & 152.64 & 4.63 & 0.0013 & 10.13 \\
\hline
\end{tabular}

\subsubsection{Rendering of radiation transfer for simulated canopies}

For each 3D stand, the Absorbed Photosynthetically Active Radiation (APAR) was computed by rendering the forest scene with a path tracing algorithm using a modified version of the PBRT path ray tracer [21]. In principle PBRT generates paths that begin at the source and propagate into the scene, eventually ending at the camera. At each vertex along a path there is a scattering event, based on the Bidirectional Scattering Distribution Function (BSDF) of the surface, from which a new path is propagated [21]. Direct and scattered irradiance assimilation was simulated by assigning a light source for each angular sun position during the period of the day of June $15^{\text {th }} 2004$. Diffuse irradiance absorbance was simulated by using a homogeneous skymap (i.e., constant illumination from each spherical direction) of which the horizontal irradiance equals the mean of all recorded diffuse irradiances during the period of one day. Light interception was calculated by placing a virtual camera 
on the surface of each leaf. Each camera recorded the intercepted irradiance onto the leaf surface as the sum of direct, diffuse, and first order scattering irradiance from neighbour leaves. To exclude edge effects, light interception computations were limited to the nine central trees of each canopy stand. Leaves were assigned a constant reflectance and transmittance of $6.5 \%$ respectively in the PAR (400-700 nm) based on measurements with an ASD spectroradiometer and a leaf probe. These assigned values are in agreement with the results obtained by [22]. Leaves were assumed to be Lambertian scatterers. The forest scene illumination input data were obtained from Meteosat data (www.satel-light.com) for a partly cloudy day (June $15^{\text {th }}$ 2004) with hourly values and sun positions for Brussels, Belgium (latitude $50.85^{\circ}$, longitude $4.37^{\circ}$ ). The rendering mechanism creates the possibility to study the impact of actual crown geometry, leaf distributions, leaf angle distributions, etc. on the canopy's light environment.

Figure 2. Simulated Fagus sylvatica (L.) stand comprised of 9 trees. Four LAI scenarios are presented. Upper stands (9 years old Fagus) are derived from in situ measured trees; (A) with the observed plantation distance of $1.8 \mathrm{~m} \times 2.0 \mathrm{~m}(\mathrm{LAI}=0.58)$, and $(\mathrm{B})$ with a changed plantation distance of $1.0 \mathrm{~m} \times 1.0 \mathrm{~m}(\mathrm{LAI}=1.29)$. Bottom stands $(15$ years old Fagus) are derived from AMAP library and have an LAI of 2.76 (C) and 3.65 (D) respectively. The axes of the figures indicate the stand dimensions in meters.
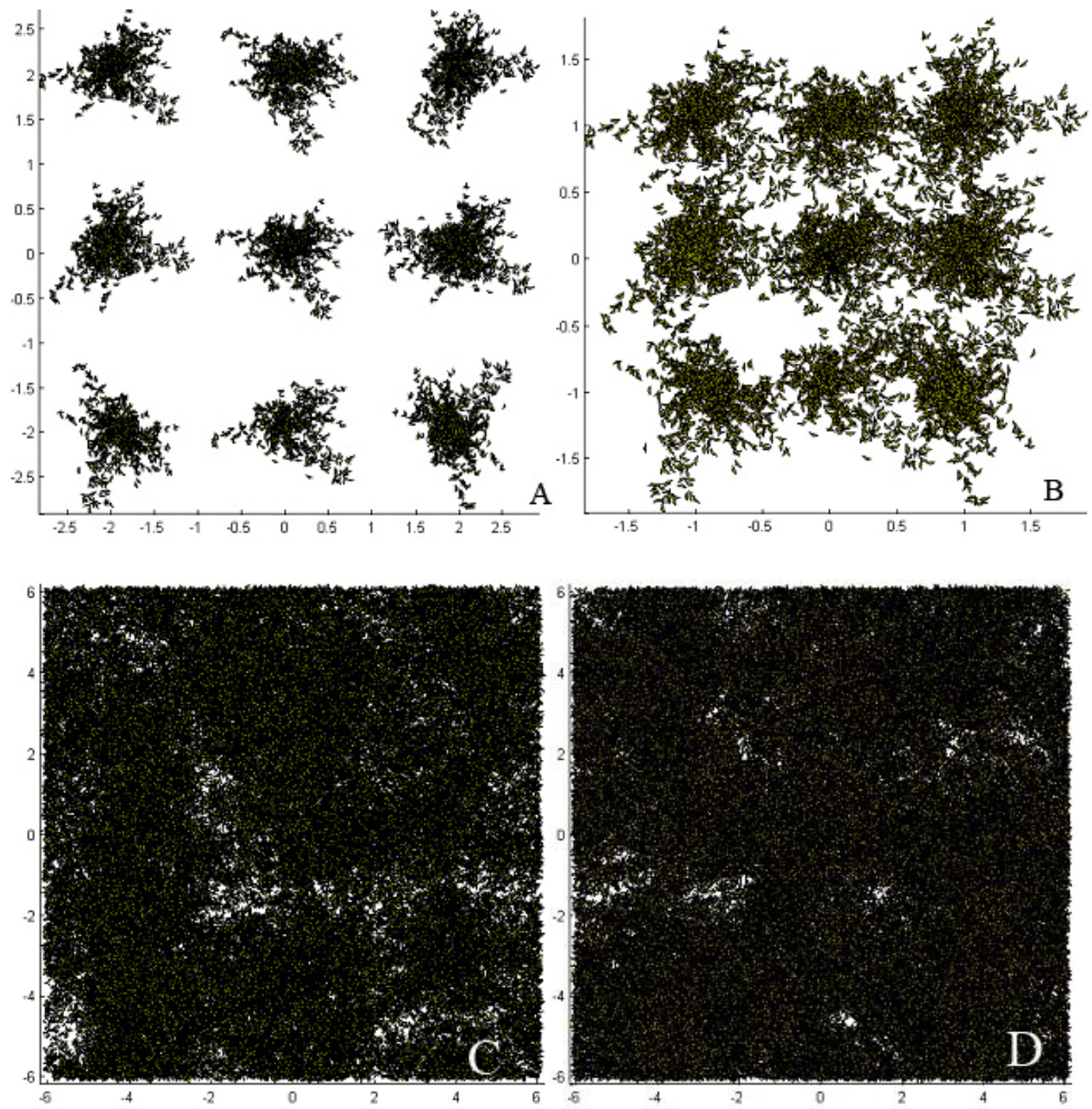


\subsection{Multilayer Canopy Models}

Three multilayer representations were created with different Leaf Normal Distribution Function (LNDF) to compare the irradiance absorbance with that of the geometrically explicit stands. The two LNDF representations (horizontal and uniform) were chosen since they are widely used to model and represent canopy architecture [24,38-39]. The first representation has a horizontal LAD, and the second a uniform LAD with all leaf inclinations ranging from $0^{\circ}$ to $90^{\circ}$ equally present. Furthermore, since we had the input values of the measured LAD of leaves a third representation (planophile) was created. Each multilayer representation comprised of 6 LAI layers equal to one sixth of the total canopy LAI while reflectance and transmittance values were the same as in the values used in the PBRT ray tracing algorithm.

The distribution of light intensity intercepted by the canopy is a function of each leaf area illuminated by direct, scattered, and diffuse irradiance, the incidence angle of incoming radiation, and the solar radiation values. APAR is calculated as the sum of absorbed direct, scattered and diffuse components by the leaves. In order to calculate direct, diffuse and scattered absorbed irradiance for the multilayer models the following equations were used [24].

Irradiance absorbed by a canopy, $\mathrm{I}_{1}$, per unit ground area, is determined by integrating equation 1 over the entire canopy leaf area [40]:

$$
I_{l}=I_{l b}(L)+I_{l d}(L)
$$

The direct beam irradiance, $\mathrm{I}_{\mathrm{lb}}(\mathrm{L})$ with scattering equals to:

$$
I_{l b}(L)=\left(1-\rho_{c b}\right) k_{b}^{\prime} I_{b}(0) \exp \left(-k_{b}^{\prime} L\right)
$$

The diffuse irradiance, $\mathrm{I}_{\mathrm{ld}}(\mathrm{L})$, is:

$$
I_{l d}(L)=\left(1-\rho_{c d}\right) k_{d}^{\prime} I_{d}(0) \exp \left(-k_{d}^{\prime} L\right)
$$

The scattered direct beam irradiance, $\mathrm{I}_{\mathrm{lbs}}(\mathrm{L})$, is given by:

$$
I_{l b s}(L)=I_{b}(0)\left[\left(1-\rho_{c b}\right) k_{b}^{\prime} \exp \left(-k_{b}^{\prime} L\right)-(1-\sigma) k_{b} \exp \left(-k_{b} L\right)\right]
$$

where L is LAI, $\rho_{\mathrm{cb}}$ and $\rho_{\mathrm{cd}}$ are canopy reflection coefficients for beam and diffuse PAR respectively. $k_{b}, k_{b}$ and $k_{d}$ are the beam, beam and scattered, and diffuse and scattered PAR extinction coefficients respectively while $\sigma$ is the leaf scattering coefficient. This methodology to calculate absorbed irradiance is described analytically in [24] and involves separating the canopy in sunlit and shaded leaf fractions.

The direct beam irradiance absorbed by the sunlit leaves is unidirectional and the angle of incidence on leaves has to be considered. The absorbed direct beam irradiance is the product of the beam irradiance perpendicular to the beam and the mean cosine of the mean leaf angle (for each different multilayer representation). As a result, the irradiance absorbed by the sunlit fraction of the canopy is equal to the sum of direct-beam, diffuse and scattered-beam components. Since the shaded part of the canopy is not receiving any direct beam irradiance only the diffuse and the scattered beam are considered. The diffuse extinction coefficient, $\mathrm{k}_{\mathrm{d}}$, for a uniform multilayer representation is 0.719 [24]. The horizontal LAD has an extinction coefficient equal to 1 for all sun zenith angles as well as for 
direct and diffuse irradiance [24]. Canopy reflection coefficients were calculated using the equations from [40] and for a horizontal distribution we have:

$$
\rho_{h}=\frac{1-(1-\sigma)^{1 / 2}}{1+(1-\sigma)^{1 / 2}}
$$

While for a uniform LAD the reflection coefficient is:

$$
\rho_{c b}(\theta)=1-\exp \left[2 \rho_{h} k_{b} /\left(1+k_{b}\right)\right]
$$

Here $\theta$ is the solar zenith angle expressed in radians.

As far as diffuse irradiance is concerned, we substitute $\mathrm{k}_{\mathrm{b}}(\theta)$ with $\mathrm{k}_{\mathrm{d}}$ in equation 6 .

The extinction and reflectance coefficients for the planophile representation as well as the variables and values used for the multilayer models are presented in the appendix.

\subsection{Leaf Stratification of Modelled and Multilayer Canopies}

It is well known that in a forest stand the foliage is not distributed evenly inside the canopy. Foliage is concentrated in tree crowns and specifically around shoots [32]. To estimate this leaf distribution and compare it with the multilayer representations the number of leaves per LAI increment was calculated for the 3D stands. Furthermore, the Percentage of Above Canopy Light (PACL) per cumulative LAI was computed. PACL is the percentage of available light above any certain position inside the canopy. At the same time, the distribution of sunlit and shaded leaves was assessed for the 3D canopies based on the leaf level intercepted radiation and compared to the multilayer representations in which this distribution is given by the sunfleck penetration equation:

$$
f_{\text {Sun }}(L)=\exp \left(-k_{b} L\right)
$$

\section{Results}

\subsection{Absorbed Irradiance of Geometrically Explicit Canopies}

The diurnal path of the sun determined a continuous movement of sun flecks through the canopy. With the sun at higher zenith angles, direct irradiance was mostly intercepted by leaves at the top of the canopy, while at near nadir angles, sun flecks penetrated deeper into the stands. Furthermore, the surrounding trees intercepted larger amounts of incoming irradiance at higher sun zenith angles, than in nadir. The absorbed irradiance derived from the geometrically explicit model and the PBRT ray tracing algorithm is presented, as seen from nadir, in Figure 3 for all the stands, while the side view of the stand with LAI 1.29 and 2.76 is presented in Figure 4. Absorbed irradiance of the stands was positively correlated with LAI values for all canopy representations.

It was observed that the top layer of the leaves were mostly sunny leaves incorporating $80-100 \%$ of the available light, while the value of intercepted radiation progressively became smaller for the leaves at the bottom of the canopy (e.g., the case of the young stands) and in the middle-bottom for the older stands driven by canopy gaps. 
Figure 3. PBRT generated APAR per leaf for all 3D simulated stands. The four figures present the nadir view of the stands with LAI $0.58,1.29,2.76$, and 3.65, respectively. The leaves are separated in percentage classes of absorbed light ranging from yellow ( $80 \%-100 \%$ absorbed light) to black ( $0 \%-5 \%$ absorbed light). Sun is at $10^{\circ}$ from nadir. The axes of the figures indicate the stand dimensions in meters.
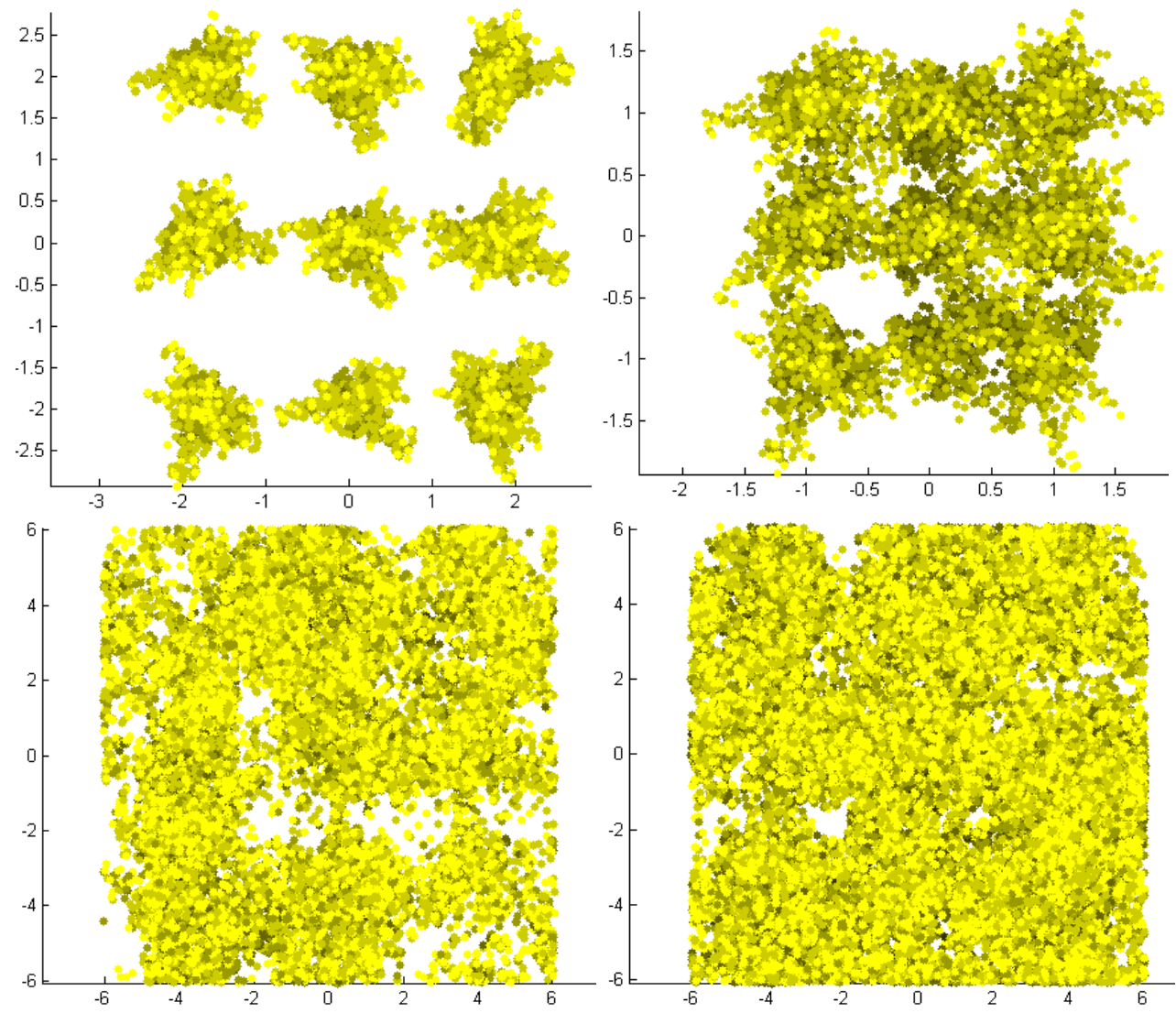

Figure 4.The two figures present the side view for the stands of LAI of 1.29, and 2.76 left and right respectively. The leaves are separated in percentage classes of absorbed light ranging from yellow ( $80 \%-100 \%$ absorbed light) to black $(0 \%-5 \%$ absorbed light). Sun is at $10^{\circ}$ from nadir. The axes of the figures indicate the stand dimensions in meters.
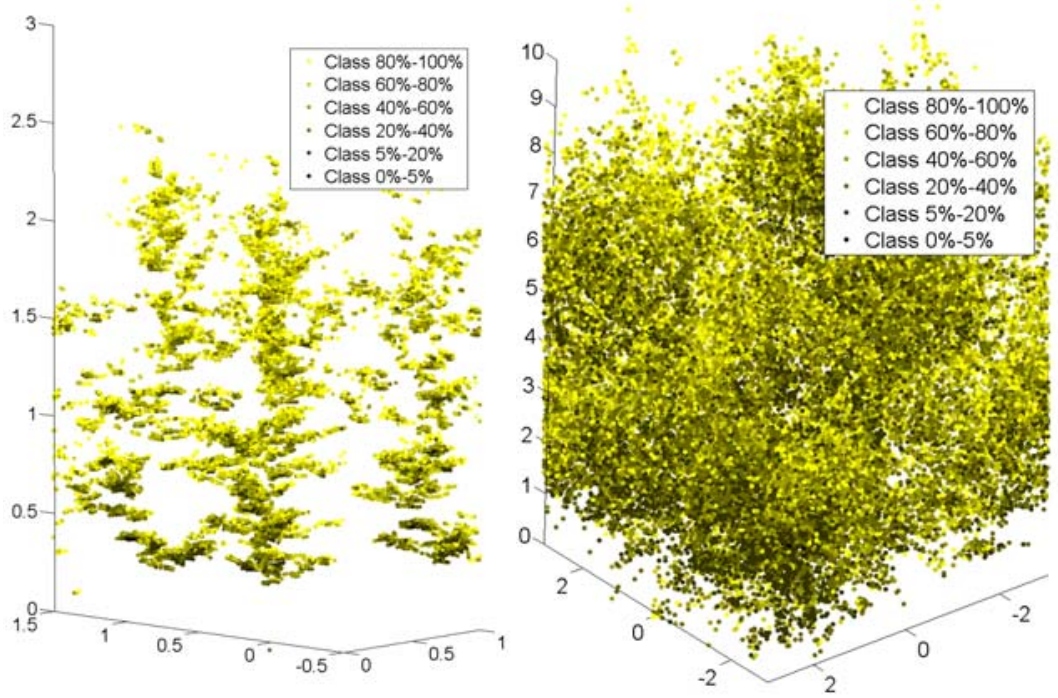


\subsection{Absorbed Irradiance of Multilayer Models}

Using the parameters for the simulated canopies and the multilayer representations set previously, the instantaneous distribution of absorbed irradiance was calculated for each sun angular position and is shown in comparison with the APAR of the 3D canopies in Figure 5 for all LAI and canopy representations. Moreover, the diurnal variation of Absorbed Photosynthetically Active Radiation irradiance is presented in Figure 6.

Figure 5. Diurnal sun angular variation of canopy APAR $\left(\mathrm{w} \mathrm{m}^{-2}\right)$. The four different graphs show absorbed irradiance from all canopy representations and LAIs.
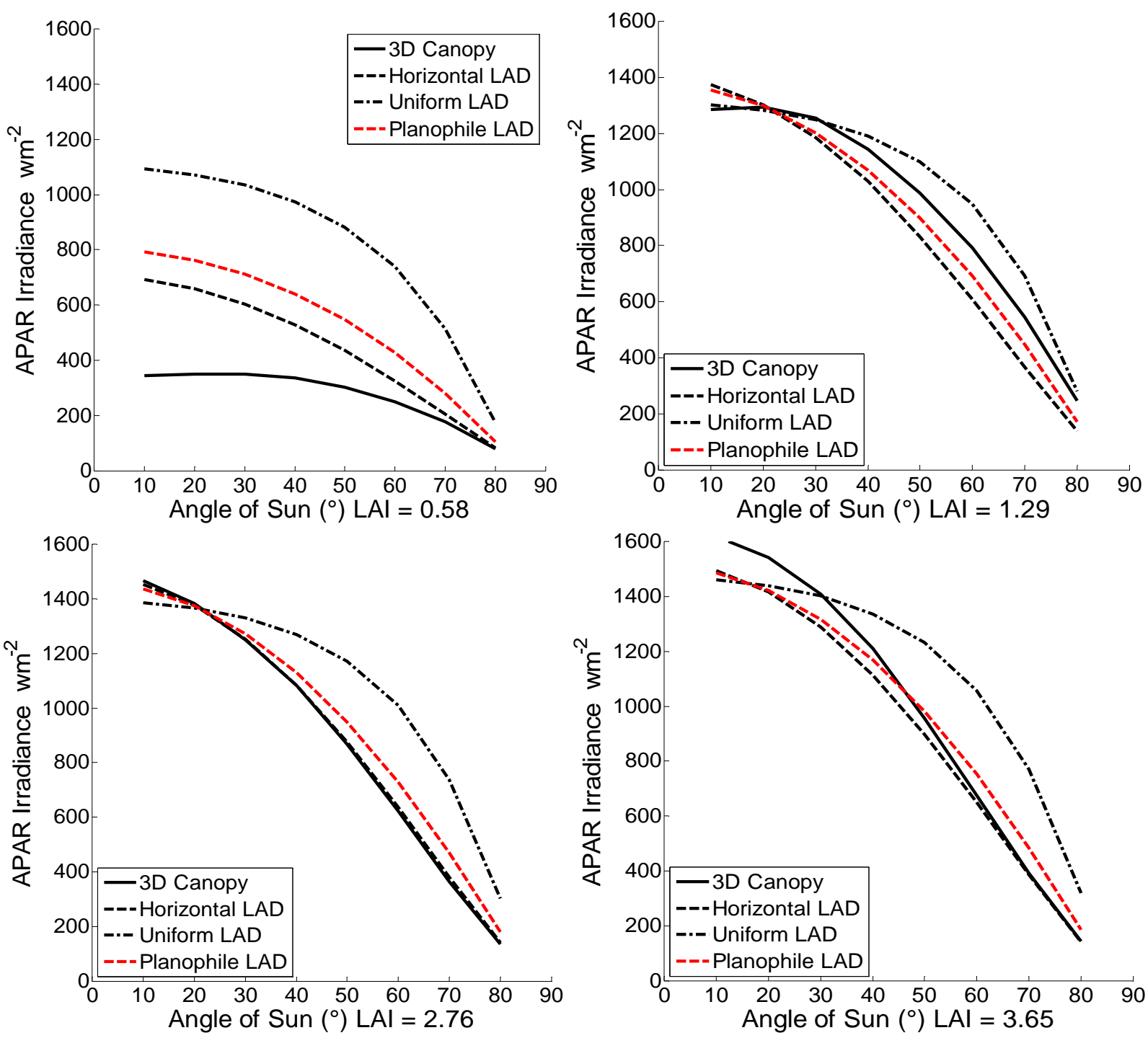
Figure 6. Diurnal variation of Absorbed Photosynthetically Active Radiation irradiance (APAR) $\left(\mathrm{w} \mathrm{m}^{-2}\right)$. Data were collected for a single day during June 2004 depicting direct, diffuse, and total irradiance for a partly cloudy sky.

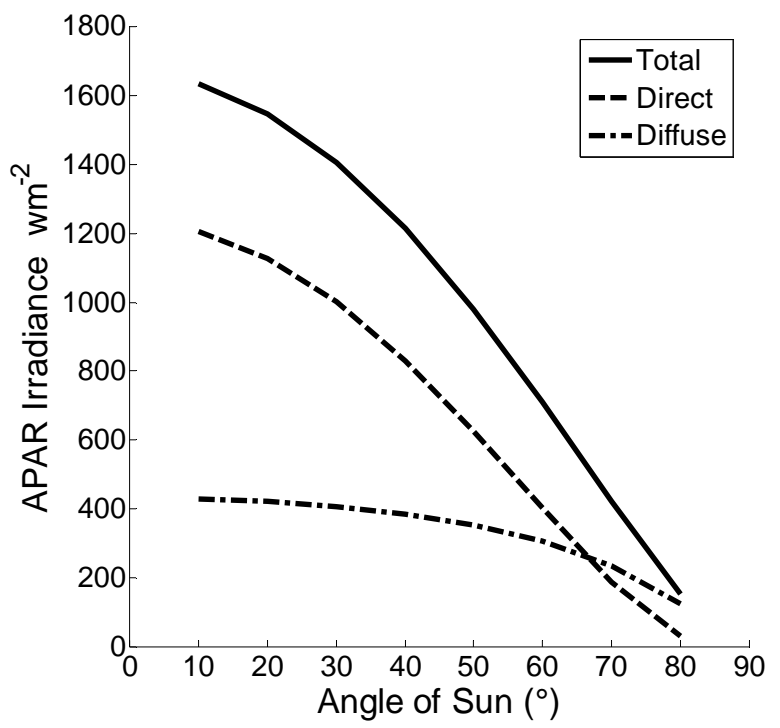

APAR values of the multilayer representations demonstrated a significant overestimation compared to the architecture measured canopy for the stand with LAI of 0.58. For the denser LAI scenario of 1.29, it was observed that the multilayer with horizontal and planophile LAD underestimated APAR while the uniform overestimated it. Compared to the geometrically explicit canopy simulated with AMAP software, it was observed that for LAI of 2.76 the horizontal and planophile LAD closely matched APAR while slightly underestimated for an increased LAI of 3.65. The uniform LAD representation in both cases overestimated the absorbed photosynthetically active radiation. The mean, maximum, and minimum APAR percentage differences between the multilayer representations and the geometrically explicit 3D canopies are presented in Table 2.

Table 2. Mean, maximum, and minimum APAR percentage differences of multilayer representations versus geometrically explicit $3 \mathrm{D}$ canopies.

\begin{tabular}{|c|c|c|c|c|c|c|c|}
\hline Stand & LAI & $\begin{array}{c}\text { Horizonta } \\
\text { l }\end{array}$ & $\begin{array}{c}\text { Max -Mean - } \\
\text { Min Values (\%) }\end{array}$ & Planophile & $\begin{array}{c}\text { Max -Mean - } \\
\text { Min Values (\%) }\end{array}$ & $\begin{array}{c}\text { Unifor } \\
\text { m }\end{array}$ & $\begin{array}{c}\text { Max -Mean - } \\
\text { Min Values (\%) }\end{array}$ \\
\hline A & 0.58 & Overest. & $101-51.77-3.76$ & Overest. & $130-86-32.8$ & Overest. & $\begin{array}{c}208-188.70- \\
120\end{array}$ \\
\hline B & 1.29 & Underest. & $44.4-17.45-6.8$ & Underest. & $\begin{array}{c}30.13-10.77- \\
0.30\end{array}$ & Overest. & $12.9-9.70-1.23$ \\
\hline $\mathbf{C}$ & 2.76 & Underest. & $3.9-1.65-0.83$ & Overest. & $\begin{array}{c}33.8-12.32- \\
0.58\end{array}$ & Overest. & $123-44.32-5.5$ \\
\hline D & 3.65 & Underest. & $7.6-5.56-1.5$ & Underest. & $29.1-11.59-2.4$ & Overest. & $121-41.45-9.8$ \\
\hline
\end{tabular}

For the sparse canopy scenario of LAI 0.58 , the representation with horizontal LAD reached differences of $101 \%$ for near nadir sun position while the lowest differences were at high sun zenith 
angles with values of $3.76 \%$. Similarly, the uniform LAD presented the higher differences near nadir sun angles. Regarding the denser measured canopy, the horizontal LAD adequately matched the absorbed irradiance with differences of $6.80 \%$ in near nadir sun angles. These differences, however, were magnified at the highest sun zenith angles reaching $44.40 \%$. At the same time the multilayer representation with uniform LAD produced differences of $1.23 \%$ near nadir and $12.90 \%$ in higher sun angles.

Compared to the AMAP simulated canopies, the multilayer model with horizontal LAD slightly underestimated APAR in the order of $0.83 \%$ for near nadir sun angles increasing up to $3.90 \%$ absolute difference for higher sun zenith values for the case of LAI of 2.76. The uniform LAD representation overestimated APAR with differences of $5.50 \%$ in the near nadir sun angles while for higher zenith angles these differences raised up to $123.00 \%$. Similarly for LAI 3.65 the horizontal LAD multilayer model underestimated the absorbed irradiance in the near nadir angles with values of $7.60 \%$ while at higher sun angles this value dropped to $1.50 \%$. At the same time the uniform LAD representation presented higher differences for higher sun zenith angles of the order of $121.00 \%$ while for near nadir these were $9.80 \%$. As far as the planophile representation was concerned, it was observed that it followed similar patterns and values as the horizontal representation for all LAI scenarios, providing the closest APAR estimates in the LAI of 1.29 with maximum and minimum percentage differences of $30.13 \%$ and $0.30 \%$ respectively.

\subsection{Leaf Stratification inside Geometrically Explicit Stands}

A detailed analysis of the leaf distribution and its effect on APAR was performed in order to further investigate the APAR results obtained by the multilayer representations and the geometrically explicit canopies. Canopies were stratified in increments of LAI (increments of 1/6) and the number of leaves per LAI increment was calculated together with the PACL per cumulative LAI (Figure 7). This analysis was performed to compare the differences in leaf quantity, position, and irradiance absorption inside a 3D canopy to a multilayer representation.

It was observed that the multilayer representations following Beer-Lambert's law presented a smooth transition in absorbed irradiance and from their top layer had already absorbed more than $20 \%$ of the available light for the LAI case of 0.58 while the geometrically explicit canopy had absorbed merely $10 \%$. As the LAI increased to 1.29 , multilayer model with horizontal LAD absorbed almost $40 \%$ of the available light from its top layer. The same pattern was visible for the AMAP generated canopies. Similarly, the planophile and the uniform representation presented the same trend for all LAI scenarios. On the contrary, the PACL for a 3D geometrical canopy was regulated from the amount of leaves present in the various height positions.

Apparently, the distribution of an architecturally explicit canopy in terms of leaves distribution did not follow the one of the multilayer representations. The majority of the leaves were placed in the middle upper part of the trees. For example, the stand of $3.65 \mathrm{LAI}$ had a vertical profile that produced LAI increments of $0.005,0.62,1.66,1.06,0.28$, and 0.007 per two meters increment respectively, while the multilayer LAI increments were fixed at 0.60 . At the same time the distribution of sunlit and shaded leaves of multilayer representation was not consistent with the ones of explicit architecture canopies. Analytically, the percentage of shadow leaves was $0 \%$ above the top layer, $36 \%$ on the first 
increment $60 \%$ on the second, $74 \%$ on the third reaching $93 \%$ at the bottom layer of the horizontal multilayer representation for an LAI of 3.65. The explicit architecture canopy of the same LAI on the other hand presented $0 \%$ of shadow leaves on the top layer, $1.8 \%$ on the first layer, $19 \%$ on the second layer, $59 \%$ on the third, and reaching $98 \%$ on the bottom layer.

Figure 7. The PACL per cumulative LAI (increments in steps of one sixth of total LAI) is presented for the 3D architecture stands. At the same time the absorbed irradiance of the multilayer representations are depicted for the equivalent LAI increments.
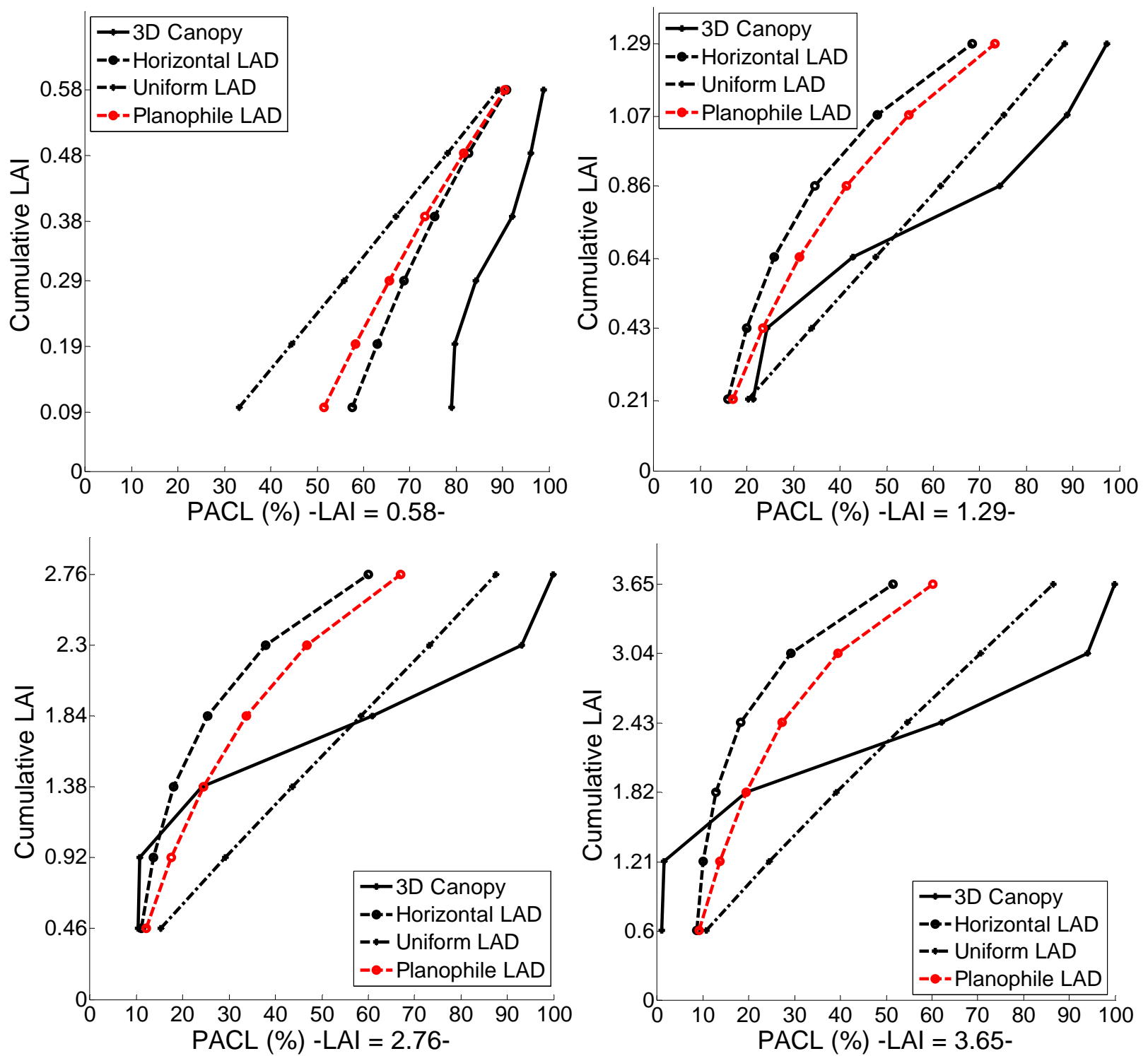

\section{Discussion}

Based on the presented results, a number of factors are identified that affect the irradiance absorbance differences between the two studied approaches. The differences on irradiance absorbance estimates among multilayer models and geometrically explicit 3D canopies for high sun zenith angles was attributed to the fact that in a dense canopy scenario the absorbed irradiance from the canopy is highly influenced by the surrounding trees. Furthermore, the clumping of the leaves near the stem and 
branches, a factor that is not integrated into the multilayer representation, highly influences APAR [32]. By comparing simulated transmissions with field measurements, [32] showed that in a homogeneous closed canopy tree leaf area is a strong indicator of irradiance absorbance, while in an open canopy location of trees and crown dimension strongly affect APAR. Moreover, [42] suggested that in a low density stand as the one of LAI of 0.58 , grouping of foliage results in a considerable decrease of APAR per unit leaf area as compared to horizontal homogeneous stands.

As far as the AMAP canopies were concerned the multilayer models exhibited significantly closer APAR values compared to the geometrically explicit canopies, a fact that was expected since they exhibit a more homogeneous and closed canopy pattern having higher LAI values. When the sun is at near nadir positions, the amount of intercepted radiation for the sum of all leaves, in the case of a dense and tall canopy, is higher than a multilayer representation due to the specific canopy leaf angle distribution and the scattering of light among the leaves themselves [42].

It was obvious that the influence of the surrounding trees regarding irradiance absorbance was higher for the AMAP simulated stand than the stand comprised from measured trees due to the difference of tree height and leaf density. As a result, higher sun zenith angles increased the percentage differences in these stands of tree height of 11 meters (LAI 2.76 and 3.65) especially for the uniform representations. These irradiance differences among the four LAI scenarios were attributed to the fact that sparse canopies have larger gaps. This fact is not considered in multilayer models. In addition, differences in tree height among the trees inside a canopy further influences irradiance absorption [43]. These observations verify that light modelling using multilayer representations of canopies are applicable for forest stands that satisfy the assumption of horizontal homogeneity, e.g., very dense stands with a closed canopy composed of only one species [38,39]. The leaf stratification results further verify these findings. Although the distribution of leaves was significantly different between the 3D canopies and multilayer models, the actual irradiance absorbance was in close agreement. Moreover, in agreement with [32,44], open stand spatial information of tree position and crown dimensions strongly affect absorption by individual leaves.

Driven by the differences observed in the high sun zenith angles, we reran the PBRT simulation of the 3D stands using solely the nine central trees (without the edge effects). It was found that for these solar angles multilayer representations provided lower maximum differences of the order of $82 \%$ for the horizontal LAD and $164 \%$ for the uniform LAD compared to the 3D canopy of LAI 0.58 . In a real case scenario for a canopy forest stand, however, the edge effect of surrounding trees is present and thus must be considered.

\section{Conclusions}

The results indicated that a multilayer representation of a stand, using a horizontal, a planophile, or a uniform modelling approach, overestimated the absorbed irradiance in a sparse canopy, while approximated the absorbed irradiance better in a closed canopy. Diurnal variation of the computed absorbed irradiance was attributed to the incidence angle of the light upon the leaves, driven by the combined effects of height and angular displacement of leaves and sun position. According to the plant density scenario and the irradiance values, the multilayer representation with horizontal and planophile LAD better fitted the 3D canopy absorbed irradiance capacity but that could be mainly attributed to the 
fact that the 3D canopy presented a planophile (closer to horizontal) distribution. It was interested though that although we simulated multilayer representations with planophile LAD based on measured values they did not provided the best fit for all the LAI cases. This fact further emphasizes the important of the role of canopy architecture in irradiance absorbance. Additionally, it was shown that the LAI distribution inside a canopy was different than the one in a multilayer representation. In the simulated canopies leaves were concentrated in the middle upper part of the trees which is not the case for the equally LAI distributed multilayer representations. Moreover, the percentage of the sunny and shadow leaves is vividly affected by the canopy distribution and does not explicitly follow Beer-Lambert's extinction law.

Several scientists developed models to investigate photon leaf absorption and photosynthetic capacity, using simulated canopies that include features such as spatial distribution of leaf area density, leaf age, and leaf inclination angles [45,46], and especially on Fagus sylvatica (L.) [4,7,43]. All the above researchers used a multilayer representation for their canopy introducing inevitably some level of structural simplification. Therefore, we wanted to present the possible deviations of these representations from a 3D case scenario. The results provide reference knowledge in the use of multilayer canopy representations, thereby acknowledging the difficulty in obtaining real tree architecture data and the necessity of simplified canopy approximations. Observation of stand characteristics under investigation in terms of LAI and plantation scheme, as well as sky irradiance characteristics, would allow scientists to fine-tune, adjust relevant parameters and incorporate bias into their multilayer representation results.

\section{Acknowledgments}

This work was funded by the OT/01/34 project of the Katholieke Universiteit Leuven. We also acknowledge the help of Jan Stuckens, from the Department of Biosystems, M3-BIORES of the Katholieke Universiteit Leuven, who assisted in the use of the PBRT ray tracing software.

\section{Appendix}

It is known that in nature leaves are almost never oriented entirely horizontally, and that they present a distribution of orientations. In order to calculate the irradiance absorbance of canopies with varying LADs, the canopy extinction coefficient should be calculated. Our measured leaves had a mean inclination angle value of $11.53^{\circ}$, while the AMAP generated trees had a mean inclination angle value of $18.24^{\circ}$. In their book [47] Campbell et al. provide an equation to calculate extinction coefficient $(\mathrm{k})$ for any possible LAD:

$$
k=\frac{\left(x^{2}+\tan ^{2} \theta\right)^{1 / 2}}{x+1.744(x+1.182)^{-0.773}}
$$

Where $\theta$ is the sun zenith angle and $\left(x=7.90\right.$ for $11.53^{\circ}$ and $x=4.80$ for $\left.18.24^{\circ}\right)$ is the leaf angle distribution parameter (mean ratio of horizontal to vertical projections of leaves). The leaf angle distribution parameter, $\mathrm{x}$, can be estimated as a function of the canopy mean leaf inclination angle $(\Theta)$ following [48] equation: 


$$
X= \begin{cases}151.515 \frac{1-0.0107 \Theta}{\Theta} & \text { if } \Theta \geq 54.4^{\circ} \\ 97.087 \frac{1-0.0053 \Theta}{\Theta} & \text { f } \Theta \leq 54.4^{\circ}\end{cases}
$$

The parameter $\mathrm{x}$ equals to 1 for a uniform distribution, zero for a vertical distribution and for a horizontal leaf canopy, $\mathrm{x}$ approaches infinity.

The diffuse transmission coefficient ( $\tau$ diff) is calculated as:

$$
\tau_{\text {diff }}=2 \int_{0}^{p i / 2} \tau_{\text {dir }}(\theta) \sin \theta \cos \theta d \theta
$$

And $\tau \operatorname{dir}(\beta)$ is the direct light transmission coefficient:

$$
\tau_{d i r}(\theta)=\exp [-k(\theta) \mathrm{L}]
$$

The above equations ( 8 to 11) along with the equations (1 to 6) were used as a reference case to create a multilayer representation with LAD similar to the measured and generated canopies $[40,41,47,48]$. The constants, variables, and the values used for the multilayer representation in Section 2.4 analysis are presented analytically in Table 3.

Table 3. Constants, variables and values used in multilayer models.

\begin{tabular}{ll}
\hline $\mathrm{L}$ & Leaf Area Index in $\mathrm{m}^{2} \mathrm{~m}^{-2}$ \\
$\rho_{\mathrm{cb}}$ & canopy reflection coefficient for beam PAR \\
$\rho_{\mathrm{cd}}$ & canopy reflection coefficient for diffuse PAR $(0.036)$ \\
$\rho_{\mathrm{h}}$ & canopy reflection coefficient of canopy with horizontal leaves $(0.0348)$ \\
$k_{b}$ & beam radiation extinction coefficient of the canopy (1 for horizontal, and $0.5 / \sin \theta$ \\
& for uniform distribution) \\
$k_{b}^{\prime}$ & beam and scattered beam PAR extinction coefficient $k_{b}^{\prime}=k_{b}(1-\sigma)^{1 / 2}$ \\
$k_{d}$ & diffuse PAR extinction coefficient (1 for horizontal and 0.78 for uniform $)$ \\
$k_{d}^{\prime}$ & diffuse and scattered diffuse PAR extinction coefficient $k_{d}^{\prime}=k_{d}(1-\sigma)^{1 / 2}$ \\
$\theta$ & solar elevation angle in radians \\
$\sigma$ & leaf scattering coefficient (reflection and transmittance) $(0.13)$ \\
\hline
\end{tabular}

\section{References and Notes}

1. Brunner, A. A light model for spatially explicit forest stand models. Ecology Man. 1998, 107, 19-46.

2. Le Roux, X.; Sinoquet, H.; Vandame, M. Spatial distribution of leaf dry weight per area and leaf nitrogen concentration in relation to local radiation regime within an isolated tree crown. Tree Physiol. 1999, 19, 181-188.

3. Epron, D.; Godard, D.; Cornic, G.; Genty, B. Limitation of net $\mathrm{CO}_{2}$ assimilation rate by internal resistances to $\mathrm{CO}_{2}$ transfer in the leaves of 2 tree species (Fagus-sylvatica L. and Castanea-sativa mill). Plant Cell Env. 1995, 18, 43-51.

4. Medlyn, B.E.; Badeck, F.W.; De Pury, D.G.G.; Barton, C.V.M.; Broadmeadow, M.; Ceulemans, R.; De Angelis, P.; Forstreuter, M.; Jach, M.E.; Kellomäki, S.; Laitat, E.; Marek, M.; Philippot, S.; Rey, A.; Strassemeyer, J.; Laitinen, K.; Liozon, R.; Portier, B.; Roberntz, P.; Wang, K.; Jstbid, 
P.G. Effects of elevated $\left[\mathrm{CO}_{2}\right]$ on photosynthesis in European forest species: a meta-analysis of model parameters. Plant Cell Env. 1999, 22, 1475-1495.

5. Röhrig, M.; Stützel, H.; Alt, C. A three-dimensional approach to modeling light interception in heterogeneous canopies. Agron. J. 1999, 91, 1024-1032.

6. Boonen, C.; Samson, R.; Janssens, K.; Pien, H.; Lemeur, R.; Berckmans, D. Scaling the spatial distribution of photosynthesis from leaf to canopy in a plant growth chamber. Ecol. Model. 2002, 156, 201-212.

7. Dufrêne, E.; Davi, H.; François, C.; Le Maire, G.; Le Dantec, V.; Granier, A. Modelling carbon and water cycles in a beech forest Part I: Model description and uncertainty analysis on modelled NEE. Ecol. Model. 2005, 185, 407-436.

8. Amthor, J.S.; Goulden, M.L.; Munger, J.W.; Wofsy, S.C. Testing a mechanistic model of forest-canopy mass and energy exchange using eddy correlation: carbon dioxide and ozone uptake by a mixed oak--maple stand. Aust. J. Plant. Physiol. 1994, 21, 623-651.

9. Bonan, G.B. A land surface model (LSM version 1.0) for ecological, hydrological, and atmospheric studies: Technical description and user's guide; NCAR Tech.: Boulder, CO, USA, 1996; p. 150.

10. Dickinson, R.E.; Shaikh, M.; Bryant, R.; Graumlich, L. Interactive canopies for a climate model. J. Climate 1998, 11, 2823-2836.

11. Monsi, M.; Saeki, T. Uber den Lichtfaktor in den Pflanzengesellschaften ubd siene Bedeutung fur die Stoffproduktion. Jap. J. Bot. 1953, 14, 22-52.

12. Myneni, R.B.; Ross, J. Photon-Vegetation Interactions. Applications in Optical Remote Sensing and Plant Ecology; Springer: Berlin, Germany, 1981; p. 565.

13. Myneni, R.B.; Ross, J.; Asrar, G. A review on the theory of photon transport in leaf canopies. Agr. Forest Meteorol. 1989, 45, 1-153.

14. Chen, S.G.; Shao, B.Y.; Impens, I.; Ceulemans, R. Effects of plant canopy structure on light interception and photosynthesis. J. Quant. Spectrosc. Radiat. 1994, 52, 115-123.

15. Planchais, I.; Sinoquet, H. Foliage determinants of light interception in sunny and shaded branches of Fagus sylvatica (L.). Agr. Forest Meteorol. 1998, 89, 241-253.

16. Flerchinger, G.N.; Yu, Q. Simplified expressions for radiation scattering in canopies with ellipsoid leaf angle distributions. Agr. Forest Meteorol. 2007, 144, 230-235.

17. Sinoquet, H.; Thanisawanyangura, S.; Mabrouk, H.; Kasemsap, P. Characterization of the light environment in canopies using 3d digitising and image processing. Ann. Bot. 1998, 82, 203-212.

18. Van der Zande, D.; Mereu, S.; Nadezhdina, N.; Cermak, J.; Muys, B.; Coppin, P.; Manes, F. 3D upscaling of transpiration from leaf to tree using ground-based LiDAR: Application on a Mediterranean Holm oak (Quercus ilex L.) tree Agr. Forest Meteorol. 2009, 149, 1573-1583.

19. Prusinkiewicz, P.; Lindenmayer, A. The algorithmic beauty of plants; Springer: Berlin, Germany, 1990; p. 228.

20. de Reffye, P.; Edelin, C.; Francon, J.; Jaeger, M.; Puech, C. Plant models faithful to botanical structure and development. Comput. Graphics 1988, 22, 151-158.

21. Pharr, M.; Humphreys, G. Physically Based Rendering from Theory to Implementation; Morgan Kaufmann: San Francisco, CA, USA, 2004; p. 860. 
22. Hosgood, B.; Jacquemoud, S.; Andreoli, G.; Verdebout, J.; Pedrini, G.; Schmuck, G. Leaf optical properties experinment (LOPEX93). In Report EUR 16095, European Commission, Joint Research Centre, Institute for Remote Sensing Applications: Ispra, Italy; 1994, p. 21.

23. Genard, M.; Baret, F.; Simon, D. A 3D peach canopy model used to evaluate the effect of tree architecture and density on photosynthesis at a range of scales. Ecol. Model. 2000, 128, 197-209.

24. De Pury, D.G.G.; Farquhar, G.D. Simple scaling of photosynthesis from leaves to canopies without the errors of big-leaf models. Plant Cell Env. 1997, 20, 537-557.

25. Raulier, F.; Bernier, P.Y.; Ung, C-H. Canopy photosynthesis of sugar maple (Acer saccharum): comparing big-leaf and multilayer extrapolations of leaf-level measurements. Tree Physiol. 1999, 19, 407-420.

26. Kucharic, J.C.; Norman M.J.; Gower, T.S. Characterization of radiation regimes in nonrandom forest canopies: theory, measurements, and a simplified modeling approach. Tree Physiol. 1999, 19, 697-706.

27. Wang, W.-M.; Li, Z.-L.; Su, H.-B. Comparison of leaf angle distribution functions: Effects on extinction coefficient and fraction of sunlit foliage. Agr. Forest Meteorol. 2007, 143, 106-122.

28. Phattaralerphong, J.; Sinoquet, H. A method for $3 \mathrm{~d}$ reconstruction of tree crown volume from photograqphs: assessment with 3d-digitized plants. Tree Physiol. 2005, 25, 1229-1242.

29. Takeda, T.; Oguma, H.; Sano, T.; Yone, Y.; Fujinuma, Y. Estimating the plant area density of a Japanese larch (Larix Kaempferi Sarg.) plantation using ground-based laser scanner. Agr. Forest Meteorol. 2008, 148, 428-438.

30. Martens, S.N.; Ustin, S.L. Measurement of tree canopy architecture. Int. J. Remote Sens. 1991, 12, 1525-1545.

31. Godin, C.; Costes, E.; Sinoquet, H. A method for describing plant architecture which integrates topology and geometry. Ann. Bot. 1999, 84, 343-357.

32. Bartelink, H.H. Radiation interception by forest trees: a simulation study on effects of stand density and foliage clustering on absorption and transmission. Ecol. Model. 1998, 105, 231-225.

33. Jonckheere, I.G.C. Consistent determination of leaf area index and quantification of foliage distribution in forest canopies based on digital hemispherical photography. Ph.D. Dissertation, Katholieke Universiteit Leuven, Leuven, Belgium, 2005; pp. 109-157.

34. Dauzat, J. Simulated plants and radiative transfer simulations. In Crop Structure and Light Microclimate. Characterization and Applications; Varlet-Grancher, C., Bonhomme, R., Sinoquet, H. Eds.; INRA: Paris, France, 1993; 271-278.

35. Saito, K.; Kumagai, Y.; Honjo, T.; Ishida, Y.; Lecoustre, R.; de Reffye, P. Photo-realistic forest landscape simulation-application of GIS and plant modeling technique-. Nicograph. Paper Contest 1993, 9, 226-236.

36. Castel, T.; Caraglio, Y.; Beaudoin, A.; Borne, F. Using SIR-C SAR data and the AMAP model for forest attibutes retrival and 3-D stand simulation. Rem. Sens. Env. 2001, 75, 279-290.

37. de Reffye, P.; Houllier, F.; Blaise, F.; Barthelemy, D.; Dauzat, J.; Auclair, D. A model simulating above- and below- ground tree architecture with agroforestry applications. In Proceedings of IUFRO 20th World Congress, Tempere, Finland, 1995; pp. 175-197. 
38. Jarvis, P.G.; Leverenz, J.W. Productivity of temperate, deciduous and evergreen forests. In Encyclopedia of Plant Physiology, N.S., Vol. 12D, Physiological Plant Ecology, IV.; Lange, O.L., Nobel, P.S., Osmond, C.B., Ziegler, H., Eds.; Springer: Berlin, Germany, 1983; pp. 233-280.

39. Wang, H.; Baldocchi, D.D. A numerical model for canopy radiation within a deciduous forest. Agr. Forest Meteorol. 1989, 46, 313-337.

40. Ross, J. The radiation regime and the architecture of plant stands. Dr. W. Junk b.v. Publishers: The Hague, The Netherlands, 1981; p. 391.

41. Goudriaan, J. The barebones of leaf-angle distribution in radiation models for canopy photosynthesis and energy exchange. Agr. Forest Meteorol. 1988, 43, 155-169.

42. Oker-Blom, P. Photosynthetic radiation regime and canopy structure in modelled forest stands. Acta For. Fenn. 1986, 197, 1-44.

43. Meir, P.; Kruijt, B.; Broadmeadow, M.; Barbosa, E.; Kull, O.; Carswell, F.; Nombre, A.; Jarvis, P.G. Acclimation of photosynthetic capacity to irradiance in tree canopies in relation to leaf nitrogen concentration and leaf mass per unit area. Plant Cell Env. 2002, 25, 343-357.

44. Cannell, M.G.T. Physiological basis of wood production: a review. Scand. J. Forest Res. 1989, 4, 459-490.

45. Field, C.B. Allocating leaf nitrogen for the maximisation of carbon gain: leaf age as a control on the allocation program. Oecologia 1983, 56, 341-347.

46. Hirose, T.; Werger, M.J.A.; Pons, T.L.; van Rheenen, J.W.A. Canopy structure and leaf nitrogen distribution in a stand of Lysimachia vulgaris L. as influenced by stand density. Oecologia 1988, 77, 145-150.

47. Campbell, G.S.; Norman, J.H. An Introduction to Environmental Biophysics, 2nd ed.; Springer: New York, NY, USA, 1998; p. 186.

48. Wang, Y.P.; Jarvis, P.G. Mean leaf angles for the ellipsoidal inclination angle distribution. Agr. Forest Meteorol. 1988, 43, 319-321.

(C) 2009 by the authors; licensee Molecular Diversity Preservation International, Basel, Switzerland. This article is an open-access article distributed under the terms and conditions of the Creative Commons Attribution license (http://creativecommons.org/licenses/by/3.0/). 